\section{Caracterização Molecular das Variantes do Carcinoma Papilífero de Tiróide}

\section{RESUMO}

Introdução e Objetivos: Muitos estudos têm demonstrado possível relação entre a expressão de oncogenes e a agressividade histológica dos tumores tiroidianos. O presente trabalho investiga as alterações moleculares existentes nas variantes do carcinoma papilífero de tiróide (CPT), através da análise da expressão de proteínas relacionadas à tumorigênese, correlacionando-a com o prognóstico. Metodologia: 32 casos estudados de CPT foram selecionados de peças cirúrgicas, entre 1987 e 2000, sendo 5 homens e 27 mulheres com idades médias de 52,6 e 46,5 anos, respectivamente. Foram estudados anticorpos monoclonais anti-MIB-1, p53, c-erb-B2, bcl-2 e receptores de TSH e TGF $\beta$. Resultados: Dos casos analisados, 11 foram considerados de melhor prognóstico (variantes microcarcinoma e encapsulado) e apresentaram elevada expressão de TSHR2 e TGFßR2 (100\%), bcl-2 (81,8\%), c-erb-B2 (90,9\%) e menor expressão de p53 e MIB-1 (18,2\%). Nos 13 casos classificados como de prognóstico intermediário (variantes clássica e folicular), a expressão de TSHR2 foi de 100\%, TGF $\beta$ R2 e bcl-2, 92,3\%; c-erb-B2, 76,9\%; p53, 23, 1\% e MIB-1, 69,2\%. Finalmente, os 8 casos de pior prognóstico (varintes de células altas e de células colunares) mostraram expressão de TSHR2, bcl-2, c-erb-B2, em 100\% dos casos, TGF $\beta$ R2 em 87,5\%, p53 em 75\% e MIB-1 em 37,5\%. Conclusão: As neoplasias mostraram-se bem diferenciadas em virtude da elevada expressão de TSHR2, TGF $\beta$ R2, e baixa de p53. Em todos os grupos, a taxa proliferativa foi ausente ou baixa $(<1,5 \%)$, com positividade maior nos tumores de prognóstico intermediário, sendo mais elevada (>1,5\%) nos carcinomas de pior prognóstico. Além disso, a positividade para a proteína bcl-2 foi progressivamente maior com a piora do prognóstico. (Arq Bras Endocrinol Metab 2003;47/3:237-242)

Descritores: Carcinoma papilífero; Imunoistoquímica; Tiróide; Prognóstico; Marcadores tumorais

\footnotetext{
ABSTRACT

Molecular Characterization of Variants of Thyroid Papillary Carcinoma. Background and objectives: Studies investigating the molecular pathogenesis of common thyroid neoplasms have shown some association between oncogenes expression and histological aggressiveness of thyroid tumors. We have investigated molecular changes in variants of thyroid papillary carcinoma (TPC), by analyzing the expression of proteins related to its tumorigenesis, and further, its own relationship to the prognosis. Methods: We examined 32 surgical specimens of TPC obtained from 5 males and 27 females (mean ages of 52.6 and 46.5 years, respectively). Monoclonal antibodies anti-MIB-1, p53, c-erb-B2, TSH receptors, TGF $\beta$ receptors and bcl-2 were detected by immunohistochemistry. Results: 11 of the 32 cases had a better prognosis (microcarcinoma and encapsulated tumors), with high expression of TSHR2 and TGF $\beta$ R2 (100\%), bcl-2 (81.8\%), c-erb-B2 (90.9\%) and lesser expression of p53 and MIB-1 (18.2\%). In the 13 cases with intermediate prognosis (classic and folicular), the expression of TSHR2 reached 100\%; TGF $\beta$ R2 and bcl-2, 92.3\%; cerb-B2, 76.9\%; p53, 23.1\%; and MIB-1, 69.2\%. The 8 cases with the worst
}

artigo original

\author{
Marcus Aurelho de Lima \\ Lauro Cézar Maito Filho \\ Helton Carlos de Almeida \\ Marlene Cabrine dos Santos \\ Maria de Fátima Borges
}

Disciplinas de Endocrinologia e Bioestatística da Faculdade de Medicina do Triângulo Mineiro, Uberaba, $M G$.

Recebido em 09/09/02 Revisado em 02/01/03 Aceito em 02/04/03 
prognosis (tall and columnar cells) expressed TSHR2, bcl-2, c-erb-B2, in 100\% of them; TGF $\beta R 2$ in 87.5\%; p53 in 75\%; and MIB-1 in 37.5\%. Conclusions: These neoplasias have shown to be well differentiated, due to the high expression of TSH and TGF $\beta$ receptors, and low expression of p53. In all groups, the proliferation rate was absent or very low (<1.5\%), with high positives in the group of intermediate prognosis. Proliferation rate was higher among the most aggressive variants. Furthermore, the positivity to bcl-2 was highly related to the worsening of prognosis. (Arq Bras Endocrinol Metab 2003;47/3:237-242)

Keywords: Papillary carcinoma; Immunohistochemistry; Thyroid cancer; Prognosis; Tumor markers

O TUMORES TIROIDIANOS têm sido pouco estudados do ponto de vista da expressão de oncogene, e isto se deve provavelmente à baixa incidência e mortalidade desta doença (1). Por outro lado, as variedades de carcinoma papilífero foram caracterizadas puramente do ponto de vista morfológico, e quando se investiga fator prognóstico ou comportamento biológico desta neoplasia, analisa-se o carcinoma como um todo, não especificando diferenças existentes no nível molecular de suas variedades. Nos últimos anos, as alterações em vários oncogenes, genes supressores de tumores e seus produtos correspondentes têm sido estabelecidos como achados importantes do processo neoplásico (2). Diversos relatos têm chamado a atenção para as alterações moleculares durante a tumorigênese da tiróide (3), mas poucos estudos têm comprovado o valor prognóstico de tais marcadores nas variáveis clinicopatológicas mais convencionais (3). Entretanto, mais recentemente foram observadas evidências de expressão aumentada e/ou alterada de oncogenes, genes supressores de tumores e seus produtos nas neoplasias tiroidianas $(5,6)$. Muitos estudos têm investigado possível relação entre a expressão do oncogene e a agressividade histológica dos tumores tiroidianos, geralmente acompanhada por rápida proliferação celular (1) e, no decorrer destes últimos anos, muito tem sido feito para elucidar o papel dos fatores de crescimento e de oncogenes no desenvolvimento e na manutenção das neoplasias tiroidianas.

Já foram analisados vários oncogenes e fatores de crescimento em carcinomas papilíferos de tiróide. Porém, esses trabalhos estudaram os carcinomas papilíferos de um modo geral sem se preocupar em investigar isoladamente a expressão dessas proteínas nas diferentes variedades deste carcinoma. As proteínas que já foram estudadas são p53, c-erbB-2, receptor do hormônio estimulador da tiróide (TSHR2), fator de cresci- mento epidérmico (EGF), fator de crescimento e transformação $\beta$ (TGF- $\beta$ ), o antígeno Ki-67 (MIB-1) e bcl-2.

A proteína p53 é uma fosfoproteína que tem papel na regulação da proliferação celular normal (7), atuando no controle do ciclo celular, reparação e síntese de DNA, na diferenciação celular, plasticidade genômica e na morte celular programada (8-10). Esta proteína aumenta à medida que aumenta o dano genômico, e acredita-se que o curso clínico benigno de tumores tiroidianos bem diferenciados (carcinoma papilífero) esteja associado à função normal de p53 (11). A proteína tem papel importante na progressão de carcinomas bem diferenciados para indiferenciados (12) e é rara em carcinomas bem diferenciados. Há correlação direta entre mutação de p53 e pior prognóstico (13).

O oncogene c-erbB-2 é proto-oncogene que codifica proteína transmembrana (p185) que tem atividade tirosinoquinase (14). Este produto de oncogene tem homologia ao receptor do fator de crescimento epidérmico $(15,16)$ e pode ser um importante fator na iniciação ou manutenção do fenótipo neoplásico em alguns carcinomas de tiróide (17). Tem sido relatado o aumento da expressão de c-erb-B2 em neoplasias malignas desta glândula $(18,19)$ e, aproximadamente 70 a 90\% dos carcinomas papilíferos, apesar da expressão fraca, são c-erb-B2 imunopositivos (18), sendo mais freqüentes em tumores de baixo grau de malignidade (4).

O TSH é o maior regulador da função e proliferação de tirócitos $(20,21)$ e atua ligando aos receptores nas membranas destas células (22). Há relação inversa entre o nível de receptor de TSH e a agressividade de carcinomas de tiróide (23). Os carcinomas papilíferos com baixa expressão de receptor de TSH possuem tendência para serem encontrados em estágios avançados e estão associados com pior prognóstico (24).

O TGF- $\beta$ é normalmente expressado e secretado pelas células foliculares e atua como inibidor do crescimento das células da tiróide $(25,26)$. A perda da regulação da proliferação celular através do TGF- $\beta$ está correlacionada com crescimento neoplásico, induzindo células neoplásicas a escaparem do seu efeito inibidor, passo importante na carcinogênese da tiróide (27).

A atividade proliferativa no tecido tiroidiano normal e em carcinomas bem diferenciados de tiróide é muito baixa (28) e é maior nos carcinomas papilíferos de alto grau (4), mostrando relação entre o curso clínico geral e atividade proliferativa em carcinoma papilífero de tiróide (29). Os carcinomas bem diferenciados de tiróide, papilífero e folicular, mostram taxas de crescimento extremamente baixas, em relação a car- 
cinomas de mesmo grau histológico que ocorrem em outros órgãos, tais como mama, pulmão, cólon e estômago $(30,31)$. Questiona-se o modo pelo qual estes tumores de tiróide crescem, e já foi proposta a hipótese de que a inibição da apoptose estivesse implicada no desenvolvimento destas neoplasias, entretanto os estudos sobre a expressão de bcl-2, proteína inibidora de apoptose, em neoplasias de tiróide (32) não mostram resultados satisfatórios, inclusive em comparação com tecidos tiroidianos não neoplásicos (33) e normais (31), não havendo diferença significativa entre as porcentagens de positividade nas categorias estudadas. Estes achados sugerem que bcl-2 não deve ter papel importante na patogenia destas lesões.

A atividade proliferativa pode ser avaliada imunoistoquimicamente usando o anticorpo monoclonal para o antígeno Ki-67 (MIB-1), que marca proteína não histona presente no núcleo das células em ciclo (34). Assim sendo, o índice de proliferação é expresso pela porcentagem de células positivas para Ki67 em cada tumor. Em outros estudos, tem-se observado que quanto mais indiferenciado o carcinoma tiroidiano, maior a porcentagem de células positivas Ki-67 e, portanto, maior o índice proliferativo (16).

\section{MATERIAL E MÉTODOS}

\section{Seleção de casos}

Foram estudadas tiróides com carcinoma papilífero, no período de 1987 a 2000 , sendo 5 homens e $27 \mathrm{mu}-$ lheres com idade média de 52,6 e 46,5 anos, respectivamente, provenientes dos arquivos do Laboratório de Anatomia Patológica na Associação de Combate ao Câncer do Brasil Central, Uberaba, MG.

Os diagnósticos de carcinoma papilífero e suas variedades foram fundamentados na morfologia histológica clássica de acordo com os critérios de Hedinger e cols. (35) e modificados por Rosai e cols. (36).

\section{Técnicas Imunoistoquímicas}

Para análise imunoistoquímica, foram utilizados os seguintes anticorpos:

l) anticorpo monoclonal contra uma parte recombinante do antígeno Ki-67 (MIB-1; Immunotech SA, Marselha, França) a uma diluição de 1:100.

2) anticorpo monoclonal contra a oncoproteína p53 (DAKO, Carpinteria, CA, USA - M7001), a uma diluição de 1:50.

3 ) anticorpo monoclonal contra a oncoproteína c-erb-B2 (DAKO, Carpinteria, CA, USA - A485), a uma diluição de 1:80.
4) anticorpo monoclonal anti-receptor do fator de crescimento e transformação $\beta$ II (TGF $\beta$ RII Santa Cruz Biotechnology, Santa Cruz, Califórnia, USA - SC220), a uma diluição de 1:100.

5) anticorpo monoclonal anti-receptor de TSH (NOVOCASTRA, Inglaterra - NCL-TSHRII), a uma diluição de 1:20.

6) anticorpo monoclonal contra a proteína bcl-2 (DAKO, Glostrup, Dinamarca), a uma diluição de 1:80.

Os tecidos foram fixados em formalina a $4 \%$, incluídos em parafina e obtidos cortes de $4 \mu \mathrm{m}$. Os cortes foram desparafinizados e hidratados. A recuperação antigênica foi feita com solução tampão de citrato de sódio $0,01 \mathrm{M}, \mathrm{pH}=6,0 \mathrm{em}$ forno microondas durante 2 ciclos de $9 \mathrm{~min}$ em potência máxima $(900 \mathrm{~W})$. Em seguida, as lâminas foram mantidas no interior do forno desligado por $20 \mathrm{~min}$ e, posteriormente, em solução de $\mathrm{H}_{2} \mathrm{O}_{2}$ a $3 \%$ em metanol por $10 \mathrm{~min}$, sendo lavadas em PBS por mais 10min. Os cortes foram incubados com os anticorpos primários nas diluições citadas anteriormente durante $22 \mathrm{~h}$, a $4^{\circ} \mathrm{C}$. Como sistema de detecção foi utilizado o LSAB - plus - peroxidase (DAKO, Carpinteria, CA, USA). A revelação foi feita utilizando-se como cromógeno a 3amino-9-etilcarbazol 0,048\% (Sigma CO., St. Louis, MO, USA - A5754) e como substrato da enzima, a $\mathrm{H}_{2} \mathrm{O}_{2}$ a $0,0097 \%$. Os cortes foram contracorados com hematoxilina de Harris.

\section{Análise das lâminas}

As lâminas preparadas foram analisadas de formas quantitativa e qualitativa. A análise qualitativa baseouse na comparação da coloração das lâminas estudadas com controles sabidamente positivos e negativos para determinados marcadores. Quantitativamente, a positividade das células tumorais foi dividida em 3 grupos, sendo estes classificados em difusamente positivos, múltiplos focos de positividade e foco isolado positivo. Em relação ao MIB-1, o percentual de células positivas (taxa de proliferação) foi avaliado observando-se grupos de 1000 células tumorais.

O estudo está de acordo com a Declaração de Helsinki de 1975, revista em 1983, e foi aprovado pelo Comitê de Ética Médica em Pesquisa da Faculdade de Medicina do Triângulo Mineiro.

\section{RESULTADOS}

\section{Variantes de melhor prognóstico}

Onze casos foram considerados de melhor prognóstico (variantes microcarcinoma e encapsulado) e apre- 
sentaram elevada expressão de TSHR2 (100\% difusamente positivos), TGF $\beta$ R2 (90,9\% difusamente positivos e $9,1 \%$ com múltiplos focos positivos), bcl-2 (54,5\% difusamente positivos; $27,3 \%$ com múltiplos focos positivos e negatividade em apenas $18,2 \%$ dos casos) e c-erb-B2 (81,8\% difusamente positivos; $9,1 \%$ com foco isolado positivo e $9,1 \%$ negativos). Expressões menores foram encontradas em p53 $(9,1 \%$ com múltiplos focos positivos; $9,1 \%$ com foco isolado positivo e $81,8 \%$ negativos) e MIB-1 (positivo em apenas 2 casos, ambos com taxa proliferativa menor que $1,5 \%)$. Os resultados estão expressos na tabela 1 .

Tabela 1. Expressão dos marcadores nas variantes, quanto ao melhor prognóstico, prognóstico intermediário e pior prognóstico do carcinoma papilífero de tiróide.

\begin{tabular}{|c|c|c|c|}
\hline & \multicolumn{3}{|c|}{ PROGNÓSTICO (\%) } \\
\hline & MELHOR & INTERMEDIÁRIO & PIOR \\
\hline \multicolumn{4}{|l|}{ TSHR2 } \\
\hline+++ & 100 & 100 & 100 \\
\hline++ & 0 & 0 & 0 \\
\hline+ & 0 & 0 & 0 \\
\hline - & 0 & 0 & 0 \\
\hline \multicolumn{4}{|c|}{ TGF $\beta R 2$} \\
\hline+++ & 90,9 & 92,3 & 87,5 \\
\hline++ & 9,1 & 0 & 0 \\
\hline+ & 0 & 0 & 0 \\
\hline - & 0 & 7,7 & 12,5 \\
\hline \multicolumn{4}{|l|}{$\mathrm{BCl}-2$} \\
\hline+++ & 54,5 & 76,9 & 100 \\
\hline++ & 27,3 & 7,7 & 0 \\
\hline+ & 0 & 7,7 & 0 \\
\hline - & 18,2 & 7,7 & 0 \\
\hline \multicolumn{4}{|c|}{ C-erb-B2 } \\
\hline+++ & 81,8 & 61,5 & 100 \\
\hline++ & 0 & 7,7 & 0 \\
\hline+ & 9,1 & 7,7 & 0 \\
\hline - & 9,1 & 23,1 & 0 \\
\hline p53 & & & 25 \\
\hline+++ & 0 & 0 & 25 \\
\hline++ & 9,1 & 7,7 & 25 \\
\hline+ & 9,1 & 15,4 & 25 \\
\hline- & 81,8 & 76,9 & \\
\hline \multicolumn{4}{|l|}{ MIB-1 } \\
\hline & $\begin{array}{c}18,2 \\
0.70\end{array}$ & $\begin{array}{c}69,2 \\
09\end{array}$ & $\begin{array}{c}37,5 \\
\text { (1) }\end{array}$ \\
\hline+ & $\begin{array}{c}\text { (0,7 a } 1 \% \text { de } \\
\text { células positivas) }\end{array}$ & $\begin{array}{c}\text { (0,1 a } 3 \% \text { de } \\
\text { células positivas) }\end{array}$ & $\begin{array}{c}\text { (1,9 a } 60 \% \text { de célu- } \\
\text { las positivas) }\end{array}$ \\
\hline- & 81,8 & 30,8 & 62,5 \\
\hline
\end{tabular}

+++ Difusamente positivo / ++ Múltiplos focos positivos /

+ Foco isolado positivo / - Negativo

\section{Variantes de prognóstico intermediário}

Nos 13 casos classificados como de prognóstico intermediário (variantes clássica e folicular), a expressão de TSHR2 foi de $100 \%$ e difusa, TGF $\beta$ R2 de 92,3\% difusa e negativa em $7,7 \%$; bcl-2 em $76,9 \%$ difusa; $7,7 \%$ com múltiplos focos positivos, e esta mesma porcentagem para casos com focos isolados positivos e casos negativos. Para o c-erb-B2 foi verificada positividade difusa em $61,5 \% ; 7,7 \%$ tanto para positividade focal múltipla quanto para positividade focal isolada e $23,1 \%$ de negatividade. A expressão de p53 foi a menor encontrada no estudo, sendo $7,7 \%$ com múltiplos focos positivos; $15,4 \%$ com foco isolado positivo e negatividade em $76,9 \%$. A positividade para MIB-1 foi quantitativamente maior que no grupo de melhor prognóstico, apresentando positividade em 8 casos $(69,2 \%)$, porém, com taxa proliferativa maior que $1,5 \%$ em apenas 2 desses casos. Os resultados são demonstrados na tabela 1 .

\section{Variantes de pior prognóstico}

Finalmente, nos 8 casos denominados de pior prognóstico (variantes de células altas e células colunares), os marcadores TSHR2, bcl-2 e c-erb-B2 foram difusamente positivos em $100 \%$ dos casos. A expressão de TGF $\beta$ R2 mostrou-se difusa em $87,5 \%$ dos casos e negativa em $12,5 \%$. A proteína p53 mostrou-se difusamente positiva em $25 \%$ dos casos e esta mesma porcentagem foi verificada para múltiplos focos positivos, foco isolado positivo e negatividade. A positividade de MIB-1 foi de $37,5 \%$; sendo a taxa proliferativa maior que $1,5 \%$ nos 3 casos positivos. Os resultados são expressos na tabela 1 .

\section{DISCUSSÃO}

A expressão da proteína p53 foi sensivelmente maior no grupo considerado de pior prognóstico, em especial na variante de células altas. Este achado confirma resultados de outros autores, como Rüter e cols. (37), reforçando a idéia de que p53 apresenta maior expressão em carcinomas mais agressivos, com pior prognóstico.

Em todos os grupos, a proliferação celular não era observada ou quando presente em baixo índice (menor que $1,5 \%$ ), com expressão maior nos tumores de prognóstico intermediário, sendo a taxa proliferativa mais elevada (maior que $1,5 \%$ ) nos carcinomas de pior prognóstico. Esses achados sugerem que a taxa proliferativa elevada está relacionada com pior prognóstico, achado já relatado em outros estudos (34).

Em relação a bcl-2, notou-se positividade progressivamente maior com a piora do prognóstico. Basolo e cols. (38) verificaram que a expressão desta proteína foi significativamente menor nos tumores indiferenciados, relacionando-a inversamente com a taxa de apoptose. Entretanto, outros estudos que utilizaram bcl-2 não mostraram resultados satisfatórios para demonstrar papel significativo na patogenia do carcinoma papilífero de tiróide. 
Estudo feito por Hougen e cols. (18) evidenciou que a proteína c-erb-B2 é característica dos carcinomas papilíferos de tiróide, em contraste com tecidos tiroidianos não neoplásicos. Em nosso estudo, verificamos elevada expressão de c-erb-B2 e não houve diferença significativa entre os grupos prognósticos estudados.

Nesta análise, foi verificada expressão positiva de TSHR2 em 100\% dos casos, independente do grupo prognóstico. Ao contrário, no trabalho feito por Tanaka e cols. (24), observou-se que a expressão de TSHR2 seria menor nas variantes de pior prognóstico, porém, deixando claro que essa menor expressão seria concluída levando-se em conta a coloração heterogênea de diferentes áreas dentro do tumor, fato este não levado em consideração neste estudo.

Por fim, a análise imunoistoquímica revelou forte expressão de TGF $\beta$ R2 em todos os subtipos de carcinoma papilífero, sem significativa diferença entre os grupos prognósticos, em concordância com estudo feito por Lloyd e cols. (39).

De acordo com os resultados do presente estudo, em geral, o carcinoma papilífero de tiróide mostrou-se como neoplasia bem diferenciada em virtude da elevada expressão de TSHR2 e TGF $\beta$ R2 e baixa positividade de p53.

\section{AGRADECIMENTOS}

Agradecemos aos técnicos do Laboratório de Anatomia Patológica e Citopatologia ligado à Associação de Combate ao Câncer do Brasil Central, à FAPEMIG e a todos aqueles que contribuíram direta ou indiretamente para a realização deste trabalho.

Este trabalho foi apresentado no XXIII Congresso Brasileiro de Patologia, realizado em Salvador, Bahia, no período de 06 a 10 de junho de 2001.

\section{REFERÊNCIAS}

1. Romano Ml, Grattone M, Karner MP, Moiguer S, Tetelbaum F, Romano LA, et al. Relationship between the level of c-myc mRNA and histologic aggressiveness in thyroid tumors. Horm Res 1993;39:161-5.

2. Wynford-Thomas D. Oncogenes and anti-oncogenes: the molecular basis of tumour behaviour. J Pathol $1991 ; 165: 187-201$

3. Farid NR, Shi Y, Zou M. Molecular basis of thyroid cancer. Endocr Rev 1994;15:202-32.

4. Akslen LA, Varhaug JE. Oncoproteins and tumor progression in papillary thyroid carcinoma. Cancer 1995;76:1643-54.
5. Aasland R, Lillehaug J, Male R, Josendal O, Varhaug JE, Kleppe K. Expression of oncogenes in thyroid tumours: co-expression of C-erbB2/neu and C-erbB2. Br J Cancer 1988;57:358-63.

6. Wyllie FS, Lemoine NR, Williams ED, Wynford-Thomas D. Structure and expression of nuclear oncogenes in multistage thyroid tumorigenesis. Br J Cancer 1989;60:561-5.

7. Finlay CA, Hinds PW, Levine AJ. The p53 protooncogene can act as a suppressor of transformation. Cell 1989;57:1083-93.

8. Harris CC, Hollstein M. Clinical implications of the p53 tumor-suppressor gene. N Engl J Med 1993;329:1318-27.

9. Lane DP. P53 and human cancers. Br Med Bull 1994;50:582-99.

10. Nigro JM, Baker SJ, Preisinger AC, Jessup JM, Hostetter R, Cleary $\mathrm{K}$, et al. Mutations in the p53 gene occur in diverse human tumour types. Nature 1989;342:705-8.

11. Wyllie FS, Haughton MF, Blaydes JP, Schlumberger M, Wynford-Thomas D. Evasion of p53-mediated growth control occurs by three alternative mechanisms in transformed thyroid epithelial cells. Oncogene 1995; 10:49-59.

12. Holm R, Nesland JM. Retinoblastoma and p53 tumor suppressor gene protein expression in carcinomas of the thyroid gland J Pathol 1994; 172:267-72.

13. Simon D, Goretzki PE, Gorelev V, Ebling B, Reishaus E, Lyons J, et al. Significance of p53 in human thyroid tumors. World J Surg 1994; 18:535-40.

14. Akiyama T. C-erb-B2 gene: a 185-kilodalton glycoprotein with thyrosine-kinase activity. Science 1986;232:1644-6.

15. Nakano T, Oka K, Ishikawa A, Morita S. Correlation of cervical carcinoma c-erbB-2 oncogene with cell proliferation parameters in patients treated with radiation therapy for cervical carcinoma. Cancer 1997;79:513-20.

16. Yamamoto T, Ikawa S, Akiyama T, Semba K, Nomura N, Miyajima $\mathrm{N}$, et al. Similarity of protein encoded by the human c-erbB-2 gene to epidermal growth factor receptor. Nature 1986;319:230-4.

17. Frauman $A G$, Moses $A C$. Oncogenes and growth factors in thyroid carcinogenesis. Endocrinol Metab Clin North Am 1990; 19:479-93.

18. Haugen DR, Akslen LA, Varhaug JE, Lillehaug JR. Expression of c-erbB-2 protein in papillary thyroid carcinomas. Br J Cancer 1992;65:832-7.

19. Lemoine NR, Willie FS, Lillehaug JR, Staddon SL, Hughes $\mathrm{CM}$, Aasland R, et al. Absence of abnormalities of the CerbB-1 and C-erbB-2 proto-oncogene in human thyroid neoplasia. Eur J Cancer 1990;26:777-9.

20. Tanaka K, Inoue H, Miki H, Komaki K, Monden Y. Heterogeneous distribution of thyrotropin receptor messenger ribonucleic acid (TSH-RmRNA) in papillary thyroid carcinomas detected by in situ hybridization. Clin Endocrinol $1996 ; 44: 259-67$.

21. Vassart G, Dumont JE. Thyrotropin receptor and the regulation of thyrocyte function and growth. Endocrine Reviews 1992;13:596-611.

22. Nagayama Y, Ropoport B. The thyrotropin receptor 25 years after its discovery: New insight after its molecular cloning. Mol Endocrinol 1992;6:145-56. 
23. Shi Y, Zou M, Farid NR. Expression of thyrotropin receptor gene in thyroid carcinoma is associated with a good prognosis. Clin Endocrinol 1993;39:269-74.

24. Tanaka K, Inoue H, Miki H, Masuda E, Kitaichi M, Komaki $\mathrm{K}$, et al. Relationship between prognostic score and thyrotropin receptor (TSH-R) In papillary thyroid carcinoma: immunohistochemical detection of TSH-R. Br J Cancer 1997:76:594-9

25. Colletta $G$, Cirafici AM, Di Carlo A. Dual effect of TGF-b on rat thyroid cells: inhibition of thyrotropism induced proliferation and reduction of thyroid-specific differentiation markers. Cancer Res 1989:49:3457-62.

26. Tsushima T, Arai M, Saji M, Ohba Y, Murakami H, Ohmura $\mathrm{E}$, et al. Effects of transforming growth factor beta on deoxyribonucleic acid synthesis and iodine metabolism in porcine thyroid cells in culture. Endocrinology $1988 ; 123: 1187-94$

27. Lazzereschi D, Ranieri A, Mincione $G$, Taccogna S, Nardi F, Colleta $G$. Human malignant thyroid tumors displayed reduced levels of transforming growth factor b receptor type II messenger RNA and protein. Cancer Res 1997:57:2071-6.

28. Wallin G, Backdahl M, Christensson B, Grimelius L, Auer $G$. Nuclear protein content and ki-67 immunoreactivity in non-neoplastic and neoplastic thyroid cells. Anal Quant Cytol Histol 1992; 14:296-303.

29. Shimizu T, Usuda N, Yamada T, Sugenoya A, lida F. Proliferative activity of human thyroid tumors evaluated by proliferating cell nuclear antigen/cyclin immunohistochemical studies. Cancer 1993;71:2807-12.

30. Katoh R, Bray CE, Suzuki K, Komiyama A, Hemmi A, Kawaoi A, et al. Growth activity in hyperplastic and neoplastic human thyroid determined by immunohistochemical staining procedure using monoclonal antibody MIB-1. Hum Pathol 1995;26:139-46.

31. Lima MA, Gontijo VA. Expressão da proteína bcl-2 em tiróides normais e tiroidopatias. Arq Bras Endocrinol Metab 1998:42:229-34.

32. Manetto V, Lorenzini R, Cordon-Cardo C, Krajewski S, Rosai J, Reed JC, et al. BCl-2 and bay expression in thyroid tumours. An immunohistochemical and western blot analysis. Virchows Arch 1997;430:125-30.
33. Branet F, Caryon P, Camallières $M$, Selves J, Brousset P. $\mathrm{BCl}-2$ proto-oncogene expression in neoplastic and nonneoplastic thyroid tissue. Bull Cancer 1996;83:213-7.

34. Erickson LA, Jin L, Wollan PC, Thompson GB, Van Heerden J, Lloyd RV. Expression of p27 and Ki-67 in benign and malignant thyroid tumors. Mod Pathol 1998;11:16974.

35. Hedinger C, Williams ED, Sobrin LH. Histological typing of thyroid tumours. WHO International Histological Classification of Tumours. $2^{\text {nd }}$ ed. Berlin: SpringerVerlag; 1998.

36. Rosai J, Carcangiu ML, De Lellis RA. Atlas of tumor pathology. Tumors of the thyroid gland. Washington: Armed Institute of Pathology. Fasc. 5, 1992.

37. Rüter A, Dreifus J, Jones $M$, Nishiyama $R$, Lennquist $S$. Overexpression of p53 in tall cell variants of papillary thyroid carcinoma. Surgery 1996; 120:1046-50.

38. Basolo F, Pollina L, Fontanini G, Fiori L, Pacini F, Baldanzi A. Apoptosis and proliferation in thyroid carcinoma: correlation with bcl-2 and p53 protein expression. Br J Cancer 1997;75:537-41.

39. Lloyd RV, Ferreiro JA, Jin L, Sebo TJ. TGF $\beta$, TGF $\beta$ receptors, $\mathrm{Ki}-67$, and $\mathrm{p} 27 \mathrm{Kip} 1$ expression in papillary thyroid carcinomas. Endocr Pathol 1997;8:293-300.

\section{Endereço para correspondência:}

Marcus Aurelho de Lima Disciplina de Endocrinologia Hospital Escola da Faculdade de Medicina do Triângulo Mineiro Rua Getúlio Guaritá, 130

38025-440 Uberaba, MG

Fax: (034) 312-6640

e.mail: lima@mednet.com.br 